\title{
Discovering Effect Modification in an Observational Study of Surgical Mortality at Hospitals with Superior Nursing
}

\author{
Kwonsang Lee, Dylan S. Small, Jesse Y. Hsu, Jeffrey H. Silber and Paul R. Rosenbaum \\ University of Pennsylvania, Philadelphia USA
}

\begin{abstract}
Summary. There is effect modification if the magnitude or stability of a treatment effect varies systematically with the level of an observed covariate. A larger or more stable treatment effect is typically less sensitive to bias from unmeasured covariates, so it is important to recognize effect modification when it is present. We illustrate a recent proposal for conducting a sensitivity analysis that empirically discovers effect modification by exploratory methods, but controls the family-wise error rate in discovered groups. The example concerns a study of mortality and use of the intensive care unit in 23,715 matched pairs of two Medicare patients, one of whom underwent surgery at a hospital identified for superior nursing, the other at a conventional hospital. The pairs were matched exactly for 130 four-digit ICD-9 surgical procedure codes and balanced 172 observed covariates. The pairs were then split into five groups of pairs by CART in its effort to locate effect modification. The evidence of a beneficial effect of magnet hospitals on mortality is least sensitive to unmeasured biases in a large group of patients undergoing rather serious surgical procedures, but in the absence of other life-threatening conditions, such as a comorbidity of congestive heart failure or an emergency admission leading to surgery.
\end{abstract}

\section{Superior nurse staffing, surgical mortality and resource utilization in Medicare}

Hospitals vary in the extent and quality of their staffing, technical capabilities and nursing work environments. Does superiority in these areas confer benefits to patients undergoing forms of general surgery that might be performed at most hospitals? To what extent and in what way do these factors affect the cost of surgical care? Are they a life-saving benefit or a pointless and unneeded expense in the case of relatively routine forms of surgery?

A recent study by Silber et al. (2016) sought to answer these questions using Medicare data for Illinois, New York and Texas in 2004-2006. A useful marker for superior staffing is superior nurse staffing, because there is a national voluntary accreditation program to recognize excellent nursing environments, so-called "magnet hospitals"; see Aiken, Havens and Sloane

Address for correspondence: Kwonsang Lee, Department of Statistics, The Wharton School, University of Pennsylvania, Philadelphia, PA 19104-6340 USA, E-mail: kwonlee@wharton.upenn.edu 5 Nov 2016 
(2000). Additionally, it is relatively easy to use Medicare files to determine the quantity of nurse staffing in the form of the nurse-to-bed ratio. The study compared patient outcomes at 35 magnet hospitals with nurse-to-bed ratios of 1 or more to outcomes for patients at 293 hospitals without magnet designation and with nurse-to-bed ratios less than 1. For brevity, hospitals in the first group are called magnet hospitals and those in the second group are called controls. The question being asked is: How does a patient's choice of hospital, magnet or control, affect the patient's outcomes and medical resource utilization? How consequential is this choice among hospitals and what are its consequences? There is no suggestion, implicit or otherwise, in this question that the nurses are the active ingredient distinguishing magnet and control hospitals, no suggestion that hiring nurses or changing the nurse environment would make control hospitals perform equivalently to magnet hospitals. Magnet designation marks a type of hospital, but does not identify what components are critical in distinguishing that type of hospital. Indeed, the 35 magnet hospitals had many advantages in staffing or technology: $21.5 \%$ of magnet hospitals were major teaching hospitals, as opposed to $5.7 \%$ of control hospitals; magnet hospitals had more nurses with advanced training, more medical residents per bed, and were somewhat more likely to have a burn unit, and to perform difficult forms of specialist surgery such as coronary bypass surgery and organ transplantation; see Silber et al. (2016, Table 1). Does a patient undergoing perhaps comparatively routine general surgery benefit from all of these capabilities or are they wasted on such a patient?

The distinction in the previous paragraph may be restated as follows. The counter-factual under study is: What would happen to a specific patient if that patient were treated at a hospital having the superior staffing of magnet hospitals when compared to what would happen to this same patient if treated at a control hospital? The counter-factual refers to sending the patient to one hospital or another. What would happen if patients were allocated to existing hospitals in a different way? The counter-factual does not contemplate changing the staffing at any hospital. Beds in hospitals with superior staffing are in limited supply, and it is a matter of considerable public importance that this limited resource be allocated to the patients most likely to benefit from it.

Some patients are in relatively good health and require relatively routine care; perhaps these patients receive little added benefit from magnet hospitals. Some patients are gravely ill and have poor prospects no matter what care is provided; perhaps these patients also receive little added benefit from magnet hospitals. In contrast, some patients would have poor outcomes with inferior care and would have better outcomes with superior care; perhaps these patients benefit most from treatment in a magnet hospital. Are magnet hospitals more effective for some types of patients than for others? This is the question of effect modification in our title.

Silber et al. (2016) created 25,752 matched pairs of two patients, one undergoing general 
surgery at a magnet hospital, the other at a control hospital. The two patients in a pair underwent the same surgical procedure as recorded in the 4-digit ICD-9 classification of surgical procedures, a total of 130 types of surgical procedure. Additionally, the matching balanced a total of 172 pretreatment covariates describing the patient's health prior to surgery; see Silber et

al. (2016, Table 2). Overall, Silber et al found significantly lower mortality at magnet hospitals than at control hospitals $(4.8 \%$ versus $5.8 \%$, McNemar $P$-value $<0.001)$, substantially lower use of the intensive care unit or ICU (32.9\% versus $42.9 \%)$ and slightly shorter length of stay; see Silber et al. (2016, Table 3) where costs and Medicare payments are also evaluated. Magnet hospitals had lower mortality rates while making less use of an expensive resource, the ICU.

In one analysis, Silber et al. (2016) grouped matched pairs based on an estimated probability of death that was controlled by the matching algorithm. The lowest risk patients appeared to benefit least from magnet hospitals. In contrast, the fourth quintile of risk - a high, but not the highest, quintile of risk - had both lower mortality and lower cost in magnet hospitals, whereas the highest risk quintile had lower mortality but higher cost at magnet hospitals. In brief, Silber et al. (2016) found evidence of effect modification.

Patients with very different medical problems may have similar probabilities of death. It is interesting that the effect of magnet hospitals appears to vary with patient risk, but it would be more interesting still to unpack patient risk into its clinical constituents, and to understand how the effect varies with these constituents. Clinicians do not think of patients in terms of their probability of death, but rather in terms of their specific health problems that are aggregated by the probability of death. In that sense, the examination of effect modification in Silber et al. (2016) is too limited to guide practice.

The current paper uses a recently proposed exploratory technique to unpack effect modification, combined with a confirmatory technique that examines the sensitivity of these conclusions to unmeasured biases. Is the ostensible effect larger, more stable or more insensitive to unmeasured bias for certain surgical procedure clusters or certain categories of patients defined by other health problems?

\section{Review of effect modification in observational studies}

\subsection{Notation for causal effects, nonrandom treatment assignment, sensitivity analysis}

In observational studies, it is known that certain patterns of treatment effects are more resistant than others to being explained away as the consequence of unmeasured biases in treatment assignment; see, for instance, Rosenbaum (2004), Zubizarreta et al. (2013), Stuart and Hanna (2013).

Effect modification occurs when the size of a treatment effect or its stability varies with the level of a pretreatment covariate, the effect modifier. Effect modification affects the sensitivity 
of ostensible treatment effects to unmeasured biases. Other things being equal, larger or more stable treatment effects are insensitive to larger unmeasured biases; see Rosenbaum (2004, 2005). As a consequence, discovering effect modification when it is present is an important aspect of appraising the evidence that distinguishes treatment effects from potential unmeasured biases, a concern in every observational study. In particular, Hsu et al. (2013, 2015) discuss sensitivity analysis in observational studies with potential effect modification, and $\oint 2$ is a concise summary. Chesher (1984), Crump et al. (2008), Lehrer, Pohl and Song (2015), Lu and White (2015), Wager and Athey (2015), Athey and Imbens (2016), and Ding et al. (2016), discuss effect modification from a different perspective, placing less emphasis on its role in confirmatory analyses that distinguish treatment effects from unmeasured biases in observational studies.

There are $I$ matched pairs, $i=1, \ldots, I$, of two subjects, $j=1,2$, one treated with $Z_{i j}=$ 1, the other control with $Z_{i j}=0$, so $Z_{i 1}+Z_{i 2}=1$ for each $i$. Subjects are matched for an observed covariate $\mathbf{x}_{i j}$, so $\mathbf{x}_{i 1}=\mathbf{x}_{i 2}=\mathbf{x}_{i}$, say, for each $i$, but may differ in terms of a covariate $u_{i j}$ that was not measured. Each subject has two potential responses, $r_{T i j}$ if treated, $r_{C i j}$ if control, exhibiting response $R_{i j}=Z_{i j} r_{T i j}+\left(1-Z_{i j}\right) r_{C i j}$, so the effect caused by the treatment, $r_{T i j}-r_{C i j}$ is not seen from any subject; see Neyman (1923) and Rubin (1974). Fisher's (1935) null hypothesis $H_{0}$ of no treatment effect asserts $r_{T i j}=r_{C i j}$ for all $i$, $j$. Simple algebra shows that the treated-minus-control pair difference in observed responses is $Y_{i}=\left(Z_{i 1}-Z_{i 2}\right)\left(R_{i 1}-R_{i 2}\right)$ which equals $\left(Z_{i 1}-Z_{i 2}\right)\left(r_{C i 1}-r_{C i 2}\right)= \pm\left(r_{C i 1}-r_{C i 2}\right)$ if Fisher's hypothesis $H_{0}$ is true. Write $\mathcal{F}=\left\{\left(r_{T i j}, r_{C i j}, \mathbf{x}_{i j}, u_{i j}\right), i=1, \ldots, I, j=1,2\right\}$ for the potential responses and covariates, and write $\mathcal{Z}$ for the event that $Z_{i 1}+Z_{i 2}=1$ for each $i$.

In a randomized experiment, $Z_{i 1}=1-Z_{i 2}$ is determined by $I$ independent flips of a fair coin, so $\pi_{i}=\operatorname{Pr}\left(Z_{i 1}=1 \mid \mathcal{F}, \mathcal{Z}\right)=\frac{1}{2}$ for each $i$, and this becomes the basis for randomization inferences, for instance for tests of Fisher's null hypothesis or for confidence intervals or point estimates formed by inverting hypothesis tests. A randomization inference derives the null distribution given $(\mathcal{F}, \mathcal{Z})$ of a test statistic as its permutation distribution using the fact that the $2^{I}$ possible values of $\mathbf{Z}=\left(Z_{i 1}, Z_{i 2}, \ldots, Z_{I 2}\right)$ each have probability $2^{-I}$ in a randomized paired experiment; see Fisher (1935), Lehmann and Romano (2005, §5), or Rosenbaum (2002a, §2). A simple model for sensitivity analysis in observational studies says that treatment assignments in distinct pairs are independent but bias due to nonrandom treatment assignment may result in $\pi_{i}$ that deviate from $\frac{1}{2}$ to the extent that $1 /(1+\Gamma) \leq \pi_{i} \leq \Gamma /(1+\Gamma)$ for $\Gamma \geq 1$, and the range of possible inferences is reported for various values of $\Gamma$ to display the magnitude of bias that would need to be present to materially alter the study's conclusion; see, for instance, Rosenbaum (2002a, $\S 4.3 .2 ; 2002 \mathrm{~b}$ ) for the case of matched binary responses, as in the current paper. For instance, a sensitivity analysis may report the range of possible $P$-values or point estimates that are consistent with the data and a bias of at most $\Gamma$ for several values of $\Gamma$. 
For various approaches to sensitivity analysis in observational studies, see Cornfield et al. (1959), Gastwirth (1992), Gilbert et al. (2003), Egleston et al. (2009), Hosman et al. (2010) and Liu et al. (2013).

\subsection{Three strategies examining effect modification}

There is effect modification if the magnitude of the effect, $r_{T i j}-r_{C i j}$, varies systematically with $\mathbf{x}_{i}$. Let $\mathcal{G}$ be a subset of the values of $\mathbf{x}$, and define the null hypothesis $H_{\mathcal{G}}$ to be Fisher's null hypothesis for individual $j$ in set $i$ with $\mathbf{x}_{i j} \in \mathcal{G}$, so $H_{\mathcal{G}}$ asserts that $r_{T i j}=r_{C i j}$ for all $i j$ with $\mathbf{x}_{i j}=\mathbf{x}_{i} \in \mathcal{G}$. Let $\mathfrak{g}=\left(\mathcal{G}_{1}, \ldots \mathcal{G}_{G}\right)$ be a mutually exclusive and exhaustive partition of values of $\mathbf{x}_{i j}=\mathbf{x}_{i}$, so each pair $i$ has an $\mathbf{x}_{i}$ contained in exactly one $\mathcal{G}_{g}$. A simple form of effect modification occurs if $H_{\mathcal{G}_{g}}$ is true for some $g$ but not for other $g$. Write $I_{g}$ for the number pairs with $\mathbf{x}_{i} \in \mathcal{G}_{g}$, so $I=\sum_{g=1}^{G} I_{g}$.

There are three strategies for defining the groups, $\mathfrak{g}=\left(\mathcal{G}_{1}, \ldots, \mathcal{G}_{G}\right)$, two of which are practically useful but technically straightforward, the third having interesting technical aspects that we illustrate using the Medicare example. One useful strategy defines the groups, $\mathfrak{g}=\left(\mathcal{G}_{1}, \ldots, \mathcal{G}_{G}\right)$, a priori, without reference to data. For example, on the basis of clinical judgement, one might believe certain surgical procedures are more challenging or hazardous than others, and therefore divide the exactly matched procedures into a few groups based on clinical judgement alone. Alternatively, clinical judgement might separate patients with severe chronic conditions unrelated to the current surgery, such as congestive heart failure.

A second strategy uses an external source of data to define the groups. In particular, Silber et al. (2016) fit a logit model to an external data source, predicting mortality from covariates, $\mathbf{x}_{i j}$, then formed five groups $\mathfrak{g}=\left(\mathcal{G}_{1}, \ldots, \mathcal{G}_{5}\right)$ based on this predicted risk for a given $\mathbf{x}$. This approach made no use of the mortality experience of the patients in the current study in defining the groups. A variant of the second strategy is to split one data set at random into two parts, create the groups using the first part, then analyze only the second part with these, again, externally determined groups.

In both of the first two strategies, the groups, $\mathfrak{g}=\left(\mathcal{G}_{1}, \ldots, \mathcal{G}_{G}\right)$, were determined by events external to the outcomes reported study. The second strategy makes explicit use of an external source of data, while the first strategy uses judgement that is presumably informed historically by various external sources of data. The key element in both strategies is that the groups were fixed before examining outcomes in the current study, and in that sense are unremarkable as groups, requiring no special handling because of their origin. With a priori groups, we could use any of a variety of methods to test the $G$ hypotheses $H_{\mathcal{G}_{g}}$ in such a way as to strongly control the family-wise error rate at $\alpha$, meaning that the chance of falsely rejecting at least one true $H_{\mathcal{G}_{g}}$ is at most $\alpha$ no matter which hypotheses are true and which are false. 
The third strategy that we illustrate here creates the groups, $\mathfrak{g}=\left(\mathcal{G}_{1}, \ldots, \mathcal{G}_{G}\right)$, by exploratory techniques using all of the current data, and then goes on to perform an analysis of the same data as if the groups had been determined a priori. The third strategy is designed so that it controls the family-wise error rate in a sensitivity analysis despite the data-dependent generation of $G$ particular groups from among the infinitely many ways of splitting the space of values of the observed covariates x. This strategy is discussed in detail in Hsu et al. (2015) and it entails certain restrictions on the way the groups are constructed.

A simple version of the strategy regresses $\left|Y_{i}\right|=\left|\left(Z_{i 1}-Z_{i 2}\right)\left(R_{i 1}-R_{i 2}\right)\right|$ on $\mathbf{x}_{i}$ using a form of regression that yields groups, such as CART. Note that the unsigned $\left|Y_{i}\right|$ not the signed $Y_{i}$ are used; that is, the regression does not know who is treated and who is control. The leaves of a CART tree become the groups, $\mathfrak{g}=\left(\mathcal{G}_{1}, \ldots, \mathcal{G}_{G}\right)$. The signs of the $Y_{i}$ are then "remembered," in an analysis that views the groups, $\mathfrak{g}=\left(\mathcal{G}_{1}, \ldots, \mathcal{G}_{G}\right)$, as fixed, so it resembles analyses that would have been appropriate with an a priori grouping of the type created by the first two strategies.

It is important to understand what is at issue in the third strategy; see Hsu et al. (2015) for a precise and general technical discussion. Briefly if obscurely, the groups, $\mathfrak{g}=\left(\mathcal{G}_{1}, \ldots, \mathcal{G}_{G}\right)$, and hence the hypotheses, $H_{\mathcal{G}_{g}}$, are not stable. If the observed data had been slightly different, the CART tree would have been different, and we would be testing different hypotheses. What does it mean to speak about the probability of falsely rejecting $H_{\mathcal{G}_{g}}$ if most data sets would not lead us to test $H_{\mathcal{G}_{g}}$ ?

Consider the simplest case, a paired randomized experiment. If Fisher's null hypothesis of no effect of any kind were true, then $Y_{i}=\left(Z_{i 1}-Z_{i 2}\right)\left(r_{C i 1}-r_{C i 2}\right)= \pm\left(r_{C i 1}-r_{C i 2}\right)$ and, given $(\mathcal{F}, \mathcal{Z})$, different random assignments $Z_{i j}$ always yield $\left|Y_{i}\right|=\left|r_{C i 1}-r_{C i 2}\right|$, so all $2^{I}$ random assignments produce the same CART tree and the same $\mathfrak{g}=\left(\mathcal{G}_{1}, \ldots, \mathcal{G}_{G}\right)$. In other words, under $H_{0}$, the CART tree and hence $\mathfrak{g}=\left(\mathcal{G}_{1}, \ldots, \mathcal{G}_{G}\right)$ is a function of $(\mathcal{F}, \mathcal{Z})$ and not of $\mathbf{Z}$. Therefore, under $H_{0}$, the $2^{I_{g}}$ possible treatment assignments for the $I_{g}$ pairs with $\mathbf{x}_{i} \in \mathcal{G}_{g}$ each have probability $2^{-I_{g}}$, resulting in conventional permutation tests within each of the $G$ groups, tests that are conditionally independent given $(\mathcal{F}, \mathcal{Z})$ under $H_{0}$. The problem occurs because we are interested in testing not just $H_{0}$, but also individual $H_{\mathcal{G}_{g}}$ when $H_{0}$ is false because some individuals are affected by the treatment. If $H_{0}$ is false, different random assignments $\mathbf{Z}$ yield different $\left|Y_{i}\right|$, hence different CART trees and different hypotheses, $\mathfrak{g}=\left(\mathcal{G}_{1}, \ldots, \mathcal{G}_{G}\right)$. With a bit of care, it is possible to demonstrate two useful facts. First, if $r_{T i j}-r_{C i j}=0$ for all $i j$ with $\mathbf{x}_{i} \in \mathcal{G}_{g}$, then the conditional distribution given $\mathfrak{g}=\left(\mathcal{G}_{1}, \ldots, \mathcal{G}_{G}\right)$ and $(\mathcal{F}, \mathcal{Z})$ of the corresponding $Z_{i j}$ with $\mathbf{x}_{i} \in \mathcal{G}_{g}$ is its usual randomization distribution. In that sense, the instability of the tree over repeated randomizations has not distorted this conditional distribution of treatment assignments in groups with no treatment effect. Second, if a method is applied to test the 
$H_{\mathcal{G}_{g}}$ that would strongly control the family-wise error rate at $\alpha$ with a priori fixed groups, then conditionally given $\mathfrak{g}=\left(\mathcal{G}_{1}, \ldots, \mathcal{G}_{G}\right)$ and $(\mathcal{F}, \mathcal{Z})$, the method will reject at least one null group with probability at most $\alpha$. These two facts are extended to include sensitivity analyses in observational studies and are proved as Propositions 1 and 2 of Hsu et al. (2015). That paper also presents some reasons to hope that subsets of $\mathbf{x}_{i}$ that systematically predict $\left|Y_{i}\right|$ may identify groups in which either the magnitude of $r_{T i j}-r_{C i j}$ or its stability varies with $\mathbf{x}_{i}$.

In the current paper, we present a practical application of this third strategy.

\section{Discovering and using effect modification in the Magnet hospital study}

\subsection{Forming groups of pairs for consideration as possible effect modifiers}

The analyses here first broke and then re-paired the pairs in Silber et al. (2016) so that: (i) as in Silber et al., every pair was exactly matched for the 130 four-digit ICD-9 surgical procedure codes, (ii) the maximum number of pairs were exactly matched for an indicator of age greater than 75, congestive heart failure (CHF), emergency admission or not, and chronic obstructive pulmonary disease (COPD). Because identically the same people were paired differently, the balancing properties of the new pairs are exactly the same as reported by Silber et al. (2016, Table 2), because balancing properties refer to marginal distributions of covariates and do not depend upon who is paired with whom.

Using rpart in R, the CART tree was built using the 22,622 pairs that were exactly matched in the sense described in the previous paragraph, regressing $\left|Y_{i}\right|$ on $\mathbf{x}_{i}$, where $Y_{i}$ records the difference in binary indicators of mortality. So, the tree is essentially trying to locate pairs discordant for mortality, $\left|Y_{i}\right|=1$, on the basis of exactly matched covariates. Here, a pair is discordant if exactly one patient in the pair died within 30-days. CART was not offered all 130 exactly matched surgical procedure codes, but rather 26 mutually exclusive clusters of the 130 surgical procedures, as listed in Table 1, plus the binary covariates age $>75$, CHF, emergency admission, and COPD. The resulting tree is depicted in Figure 1. A few procedure clusters - e.g., liver procedures - are diverse, perhaps meriting further subdivision that we do not consider here.

The tree in Figure 1 did not use two variables that were considered by it, namely 'age $>75$ ' and COPD. In going to 22,622 pairs, we had omitted some pairs because they were not exactly matched for 'age $>75$ ' and COPD, but that omission is not needed to use the tree in Figure 1. Can we recover some of these omitted pairs? To recover omitted pairs, we followed the tactic in Hsu et al. (2015). Specifically, we re-paired as many of the pairs that were not used to build the tree to be exact for the 130 procedures plus CHF and emergency admission, adding these additional 1,093 pairs to the groups in Figure 1, making 23,715 pairs in total, or $95 \%$ of the original study. All analyses that follow refer to these 23,715 pairs. 
Consider the tree in Figure 1, starting from its root at the top of the figure. The tree split the population into two groups, patients without congestive heart failure $(\mathrm{CHF})$ and patients with CHF, a serious comorbid condition. It then split this divided population by grouping the 26 surgical procedure clusters. There are, of course, many way to group 26 procedure clusters; for instance, there are $2^{26}-1=67,108,863$ ways to split them into two groups. The procedure groups, proc1 and proc2, for patients without CHF are slightly different from the procedure groups, proc3 and proc4, for patients with CHF. Table 1 displays CART's grouping of the 26 procedure clusters into proc1, proc2, proc3 and proc4. In Figure 1, CART further divided proc2 into two subsets of patients, those admitted as emergencies and the remaining nonemergent patients. In Table 1, notice that proc1 and proc3 overlap extensively, as do proc2 and proc4. To the clinical eye, with a few raised eyebrows, the procedures in proc 2 and proc4 look riskier or more complex than those in proc1 and proc3. Some procedure clusters slipped. Appendectomy is grouped with the less risky procedures if the patient does not have CHF, but it grouped with the more risky procedures for a patient with CHF; however, it is unclear whether that slip is a profound insight or a hiccup.

Up to this point in the analysis, the signs of the $Y_{i}{ }^{\prime}$ 's for discordant pairs have not been used; the tree knew nothing about who lived and who died in pairs discordant for mortality.

\subsection{Informal examination of outcomes}

In 43.3 , an analysis of mortality is carried out as proposed in Hsu et al. (2015). This analysis is easier to understand if we take a quick look first. The upper part of Table 2 describes mortality informally. The first three numeric rows of Table 2 describe information that CART could use in building the tree, namely the number of pairs, the number of discordant pairs, and the proportion of discordant pairs. In Table 2, 43\% $=10127 / 23715$ of pairs are in the group 1, that is, patients without CHF undergoing less risky procedures. Expressed differently, group 1 has the most pairs and the fewest discordant pairs of the five groups. As one might expect given the information that CART was permitted to use, the proportion of discordant pairs varies markedly among the groups CART built.

The next three numeric rows of Table 2 display outcomes by treatment group, making use of $Y_{i}$ and not just $\left|Y_{i}\right|$. The mortality rates for magnet and control groups are given, as is the odds ratio computed from discordant pairs; see Cox (1970). All of the odds ratios are greater than or equal to 1 , suggesting higher mortality at control hospitals. The largest odds ratio is in group 2, 1.53, while the largest difference in mortality rates is in group 5, 18.6\%-16.5\%= $2.1 \%$. The odds ratio closest to 1 is in group 3, the group most similar to group 2 except for admission through the emergency room. 


\subsection{Structured analysis of outcomes in discovered groups}

The structured analysis in Hsu et al. (2015) starts by computing randomization tests and upper sensitivity bounds on $P$-values for each of the five groups separately. In Table 2 , these are based on a test of the McNemar type, essentially binomial calculations using discordant pairs; see Cox (1970) for discussion of paired binary data, and see Rosenbaum (2002, §4.3.2) for the sensitivity analysis. In the bottom part of Table 2 are upper bounds on one-sided $P$-values testing no treatment effect in a group in the presence of a bias in treatment assignment of at most $\Gamma$. Also given in Table 2 are the odds ratios from discordant pairs associated with McNemar's test.

The final column in the bottom of Table 2 gives the $P$-value for the truncated product of $P$ values as proposed by Zaykin et al. (2002). The truncated product generalizes Fisher's method for combining independent $P$-values: the test statistic is the product of those $P$-values that are smaller than a threshold, $\tau$, where $\tau=0.1$ in Table 2. Zaykin et al. (2002) determined the null distribution of the truncated product statistic. Hsu et al (2013) show that the same null distribution may be used to combine upper bounds on $P$-values in a sensitivity analysis for a tree like Figure 1, and that it often has superior power in this context compared to Fisher's product of all $P$-values, essentially because sensitivity analyses promise $P$-values that are stochastically larger than uniform for a given $\Gamma$. Truncation eliminates some very large upper bounds on $P$-values.

Hsu et al. (2015) combine the truncated product statistic with the closed testing procedure of Marcus et al. (1976) to strongly control the family-wise error rate at $\alpha$ in a sensitivity analysis with a bias of at most $\Gamma$. Given $G$ hypotheses, $H_{\mathcal{G}_{g}}, g=1, \ldots, G$, asserting no effect in each of $G$ groups, closed testing begins by defining $2^{G}-1$ intersection hypotheses, $H_{\mathcal{L}}$, where $\mathcal{L} \subseteq\{1, \ldots, G\}$ is a nonempty set, and $H_{\mathcal{L}}$ asserts that $H_{\mathcal{G}_{\ell}}$ is true for every $\ell \in \mathcal{L}$. Closed testing rejects $H_{\mathcal{L}}$ if and only if the $P$-value testing $H_{\mathcal{K}}$ is $\leq \alpha$ for every $\mathcal{K} \supseteq \mathcal{L}$. The $P$-value testing $H_{\mathcal{K}}$ is based on the truncated product of $P$-values for $H_{\mathcal{G}_{k}}$ for $k \in \mathcal{K}$.

The $P$-value in the final column of Table 2 tests Fisher's hypothesis $H_{0}$, or $H_{\mathcal{L}}$ with $\mathcal{L}=$ $\{1,2,3,4,5\}$. For $\Gamma=1$, this test combines five McNemar tests using the truncated product, and in the absence of bias, the hypothesis $H_{0}$ is rejected with a one-sided $P$-value of $2.7 \times 10^{-6}$. To complete closed testing of subhypotheses, one performs $2^{5}-1=31$ tests of intersection hypotheses. Hypothesis $H_{\{3,4\}}$ has a $P$-value using the truncated product of 0.080 , so neither $H_{\mathcal{G}_{3}}$ nor $H_{\mathcal{G}_{4}}$ is rejected at the 0.05 level by closed testing, but $H_{\mathcal{G}_{1}}, H_{\mathcal{G}_{2}}$ and $H_{\mathcal{G}_{5}}$ are rejected. In short, in the absence of bias, $\Gamma=1$, the hypothesis of no effect is rejected in groups 1,2 , and 5 .

At $\Gamma=1.05$, Fisher's hypothesis of no effect at all is rejected at the $9.0 \times 10^{-5}$ level, and closed testing rejects both $H_{\mathcal{G}_{1}}$ and $H_{\mathcal{G}_{2}}$ at the 0.05 level. At $\Gamma=1.10$, Fisher's hypothesis $H_{0}$ of no effect is rejected at the 0.012 level, but only $H_{\mathcal{G}_{2}}$ is rejected at the 0.05 level. At 
$\Gamma=1.17$, Fisher's hypothesis $H_{0}$ of no effect is rejected at the 0.044 level, no individual subgroup hypothesis is rejected at the 0.05 level, but $H_{\{1,2\}}$ is rejected at the 0.05 level. At $\Gamma=1.18$, no hypothesis is rejected at the 0.05 level.

A bias of $\Gamma=1.17$ corresponds with an unobserved covariate that doubles the odds of having surgery at a control hospital and increases the odds of death by more than $60 \%$. That is, stated technically, $\Gamma=1.17$ amplifies to $(\Lambda, \Delta)=(2.0,1.61)$; see Rosenbaum and Silber (2009). McNemar's test applied to all 23,715 pairs yields a $P$-value bound of 0.063 at $\Gamma=1.15$, so this overall test is slightly more sensitive to unmeasured biases and provides no information about subgroups.

\subsection{Use of the intensive care unit (ICU)}

In Table 2, magnet hospitals exhibited lower mortality than control hospitals for ostensibly similar patients undergoing the same surgical procedure, that is, magnet hospitals exhibited better quality. Does better quality cost more? For resources that are allocated by a market mechanism - say, restaurants or hotels - we expect better quality to cost more, but market forces play little role in Medicare payments. In the absence of market forces, it is an open question whether better quality costs more. Silber et al. (2016) examine this issue in several ways, but Table 3 restricts attention to the consumption of a particularly expensive resource, namely use of the intensive care unit or ICU. In a hospital with inadequate nursing staff, a patient may be placed in the ICU to ensure that the patient is monitored, while in a hospital with superior nursing this same patient might remain in a conventional hospital room. This is one mechanism by which better quality - lower mortality rates - might cost less, not more.

Is the lower mortality in magnet hospitals associated with greater use of the ICU? Apparently not. Overall and in all five groups in Figure 1, the use of the ICU in Table 3 is lower at magnet hospitals than at control hospitals. The odds ratio is largest in group 2, but it is not small in any group. In various other ways also, Silber et al. (2016) found that costs were lower at hospitals with superior nursing, despite lower mortality rates.

The closed testing procedure applied to the sensitivity analysis in the bottom part of Table 3 rejects the null hypothesis of no effect on ICU utilization in all five groups providing the bias in treatment assignment is at most $\Gamma=1.5$. Using the method in Rosenbaum and Silber (2009), a bias of $\Gamma=1.5$ corresponds with an unobserved covariate that increases the odds of surgery at a control hospital by a factor of 4 and increases the odds of going to the ICU by a factor of 2. Closed testing rejects no effect only in group 2 for $1.6 \leq \Gamma \leq 1.8$, and cannot reject even Fisher's $H_{0}$ for $\Gamma=1.9$. Detailed results for group 2 are given in Table 4 .

To emphasize a point emphasized in $§ 1$, Tables 2, 3 and 4 concern the effect of going to a magnet hospital rather than a control hospital for surgery, but they do not show the specific 
role of nurses in this effect. It is entirely plausible that superior nurse staffing would permit more patients to stay out of the ICU, but nothing in the data speaks to this directly. The main difference between the ICU and the floor of the hospital is the higher density, often higher quality, of the nurse staffing in the ICU. A hospital with a higher nurse-to-bed ratio and superior nurse staffing may be able to care for a seriously ill patient on the hospital floor, where some other hospital would be forced to send the same patient to the ICU.

\subsection{Other analyses and options for analysis}

The tree in Figure 1 was built for mortality, but was used also for ICU use. In an additional analysis, we applied CART to each leaf of Figure 1 to predict unsigned discordance for ICU use. The two interesting aspects of this analysis were: (i) subgroup 2 in Figure 1 was not further divided; (ii) subgroup 5 in Figure 1 was further divided, with more evidence of an effect on ICU use among patients in this subgroup who were not admitted through the emergency room, a pattern analogous to subgroups 2 and 3. An interesting feature of this type of analysis is that it makes mortality the primary endpoint, as it would be in most surgical studies, so only mortality determines the initial tree for the mortality analysis, but it permits the secondary outcome of ICU use to affect a secondary tree.

We let CART build the groups. Any analysis that used only $\left|Y_{i}\right|$ and $\mathbf{x}_{i}$ could be used to build the groups. In saying this, we mean that the strong control of the family-wise error rate in Hsu et al. (2015) would not be affected by revisions to the tree that used only $\left|Y_{i}\right|$ and

$\mathbf{x}_{i}$. Indeed, a surgeon who did not look at $Y_{i}$ could look at Figure 1 and Table 1 and decide to regroup some of the procedure groups. Perhaps the surgeon would view some of CART's decisions as clinically unwise and would change them, or perhaps the surgeon would prefer that proc1 and proc3 be identical, and that proc2 and proc4 be identical. Indeed, the surgeon might suggest fitting the tree again, using only $\left|Y_{i}\right|$ and $\mathbf{x}_{i}$, but subdividing some procedure clusters, say liver procedures, that seem too broad to be clinically meaningful. What is critical is that the groups are formed using $\left|Y_{i}\right|$ and $\mathbf{x}_{i}$ without using the sign of $Y_{i}$.

\section{Summary and discussion: Confirmatory analyses that discover larger effects by exploratory methods}

\subsection{Summary: It is important to notice subgroups with larger treatment effects in observa- tional studies}

In an observational study of treatment effects, there is invariably concern that an ostensible treatment effect is not actually an effect caused by the treatment, but rather some unmeasured bias distinguishing treated and control groups. Larger or more stable treatment effects are more insensitive to such concerns than smaller or more erratic effects; that is, larger biases 
measured by $\Gamma$ would need to be present to explain a large and stable treatment effect. These considerations motivate an interest in effect modification in observational studies. Perhaps the treatment effect is larger or more stable in certain subgroups defined by observed covariates. If so, the ostensible treatment effect in such subgroups is likely to be insensitive to larger unmeasured biases, therefore more credible, and additionally, a larger or more stable effect is likely to be more important clinically.

The magnet hospitals had lower mortality overall, and lower or equivalent mortality in each of the five groups. However, the superior staffing of magnet hospitals was least sensitive to unmeasured bias in our group 2, consisting of patients undergoing relatively serious forms of surgery in the absence of other life-threatening conditions, such as congestive heart failure or an emergency admission leading to surgery. Moreover, not only were mortality rates lower in magnet hospitals for these patients (2.5\% rather than $3.5 \%$ ), but additionally the magnet hospitals cared for these patients with greatly reduced use of an expensive resource, namely the intensive care unit (ICU rate of $28.9 \%$ rather than $43.3 \%$ ). Determining the cost of hospital care for Medicare patients is not straightforward, so Silber et al. (2016) contrasted several formulas to appraise the cost of magnet hospitals. In all of these formulas, use of the ICU plays a substantial part, as does the length of stay in the hospital. Regardless of which formula was used, magnet hospitals appear to produce lower mortality either at no additional cost or with a cost savings.

A plausible interpretation of Figure 1, Table 1 and Table 2 is that: (i) patients in groups 2, 4 and 5 should be directed to magnet hospitals, a limited resource; (ii) the large number of comparatively healthy patients requiring simpler surgical procedures may go to non-magnet hospitals if space in a magnet hospital is unavailable, (iii) patients in group 3 requiring emergency surgery should go to the nearest hospital.

\subsection{Exploration, confirmation or prediction using regression trees}

The CART method of Breiman, Friedman, Olshen and Stone (1984) immediately attracted the attention of clinicians, in part because its relatively compact and coarse regression trees resemble clinical thinking. Many clinical decisions - e.g., which patients should go to the ICU - are discrete choices, and distinctions that are critical in one context may be unimportant in another context, a pattern often suggested by a CART tree. For a discussion of regression trees that emphasizes its parallel with clinical thinking, see Zhang and Singer (2010). Alas, CART trees can be either unstable or too coarse or both. Subsequent work found that the much finer distinctions produced by random forests, boosting or BART improved predictions when compared with the compact trees produced by CART; see, for instance, Schapire, Freund, Bartlett, and Lee (1998), Breiman (2001a), and Chipman, George and McCulloch (2010). Although 
these much finer distinctions improved prediction, and although they have a role in improving clinical assessments, they were no longer intelligible to humans, and consequently had limited value in scientific publications in medical journals. A clinician may consider an algorithm's assessment, but the clinician cannot transfer responsibility for the patient to the algorithm, so the clinician needs to incorporate intelligible considerations in her decisions. Though not a recent development, the proverb's admonition, "Happy is the $[\ldots$ person ... ] that getteth understanding," has lost none of its force (Proverbs 3:13, King James Version). For all their faults, CART's coarse trees can be understood.

The CART method, as originally proposed, did not lend itself to conventional inference, such as hypothesis testing, much less to simultaneous inference for the groups it produced. Breiman (2001b) took pride in the gap between methods like CART and confirmatory statistical analyses of the type published in scientific journals, but it is at least debatable whether the gap is an asset or a liability.

In contrast, Hsu et al. (2015) proposed a way to use CART, or similar methods, combining exploratory construction of groups together with a confirmatory sensitivity analysis that controls the family-wise error rate in the constructed groups. All of the data are used to build the tree and all of the data are used in confirmatory analyses. This is important in Table 2 because the number of pairs discordant for mortality is not large in some groups - happily, most people survive surgery - so sample splitting to build the groups would leave less data for confirmatory analyses. The double use of all of the data works by having CART predict $\left|Y_{i}\right|$ from $x_{i}$ without knowing who is treated and who is control, then using the signed $Y_{i}$ in confirmatory analyses with CART's groups. CART trees can be unstable, so the tree should be regarded as an interesting partition of the data, not a search for a "true" partition. The formal hypothesis tests are conditional inferences given CART's partition: they correctly use, but do not endorse, the partition.

Will a tree built from $\left|Y_{i}\right|$ be useful in the study of effect modification? It is straightforward to construct theoretical examples in which an analysis of $\left|Y_{i}\right|$ would miss effect modification that an analysis of $Y_{i}$ might find. Obviously, a tree built from all of the $Y_{i}$ is preferable, but this would preclude a confirmatory analysis using the same data. As noted by Hsu et al. (2015), a result of Jogdeo (1977, Theorem 2.2) provides some encouragement. A simple version of this result says: if $Y_{i}=\mu_{i}+\epsilon_{i}, \mu_{i} \geq 0, i=1, \ldots, I$, where the errors $\epsilon_{i}$ are independent and identically distributed with a unimodal distribution symmetric about zero, then $\left|Y_{i}\right|$ is stochastically larger than $\left|Y_{j}\right|$ whenever $\mu_{i}>\mu_{j}$. Under this simple model, trees that form groups from the level of $\left|Y_{i}\right|$ have some hope of finding groups heterogeneous in $\mu_{i}$. True, if the $\epsilon_{i}$ are not identically distributed, if the dispersion of $\epsilon_{i}$ varies with $i$, then the groups may be affected by both level and dispersion; however, sensitivity to unmeasured bias is also affected 
by both the level and dispersion of the treatment effects, so groups reflecting unequal dispersion are interesting also. For additional encouragement, see also the simulation results in Hsu et al. (2015).

\subsection{Alternative methods}

As noted in 2.2 , there are three basic approaches to confirmatory sensitivity analyses for effect modification. One approach starts with a priori groups, or, what amounts to the same thing, groups built from one or more external data sets. Essentially this approach was used in Silber et al. (2016) for these data. The five groups were defined by quintiles of risk-of-death as estimated using a model fit to another set of data. That analysis was enlightening, but the plausible interpretation at the end of 4.1 makes useful distinctions that risk quintiles do not make.

Another approach is to: (i) split the data into two parts at random, (ii) form patient groups from $Y_{i}$ rather than $\left|Y_{i}\right|$ using the first part of the data, (iii) discard the first part, (iv) perform a confirmatory analysis on the second part using the patient groups formed from the first part. This approach is attractive when $I$ is very large. For some indirectly related theory, see Heller et al. (2009). Presumably, if we had twice as many pairs as we actually had, $I \rightarrow 2 I$, if we split the data in half as just described, then the resulting analysis would be uniformly better than the analysis we did with half as much data, because: (i) the tree would be better having been built from $Y_{i}$ instead of $\left|Y_{i}\right|$, but (ii) the confirmatory analysis would have the same quantity of data as our confirmatory analysis. Silber et al. (2016) used data from New York, Illinois and Texas primarily because purchasing Medicare data is expensive. There are, however, 47 more states where these came from.

\section{References}

Aiken, L. H., Havens, D. S. and Sloane, D. M. (2000) The magnet nursing services recognition program. Am. J. Nursing, 100, 26-35. http://www.nursecredentialing.org/Magnet/ProgramOverview.aspx

Athey, S. and Imbens, G. (2016) Recursive partitioning for heterogeneous causal effects. Proc. Nat. Acad. Sci., 113, 7353-7360.

Breiman, L., Friedman, J. H., Oshen, R. A., and Stone, C. J. (1984) Classification and Regression Trees. California: Wadsworth.

Breiman, L., (2001a) Random forests. Mach. Learn., 45, 5-32.

Breiman, (2001b) Statistical modeling: The two cultures (with Discussion). Statist. Sci. 16, 199-231.

Chesher, A. (1984) Testing for neglected heterogeneity. Econometrica, 52, 865-872. 
Chipman, H. A., George, E. I. and McCulloch, R. E. (2010) BART: Bayesian additive regression trees. Ann. Appl. Statist., 4, 266-298.

Cornfield, J., Haenszel, W., Hammond, E., Lilienfeld, A., Shimkin, M., Wynder, E. (1959) Smoking and lung cancer. J. Nat. Cancer Inst., 22, 173-203.

Cox, D. R. (1970) Analysis of Binary Data. London: Metheun.

Crump, R. K., Hotz, V. J., Imbens, G. W., \& Mitnik, O. A. (2008) Nonparametric tests for treatment effect heterogeneity. Rev. Econ. Statist., 90, 389-405.

Ding, P., Feller, A. and Miratrix, L. (2016) Randomization inference for treatment effect variation. J. Roy. Statist. Soc. B 78, 655-671.

Egleston, B. L., Scharfstein, D. O. and MacKenzie, E. (2009) On estimation of the survivor average causal effect in observational studies when important confounders are missing due to death. Biometrics, 65, 497-504.

Fisher, R. A. (1935) The Design of Experiments. Edinburgh: Oliver \& Boyd.

Gastwirth, J. L. (1992) Methods for assessing the sensitivity of statistical comparisons used in Title VII cases to omitted variables. Jurimetrics, 33, 19-34.

Gilbert, P., Bosch, R., Hudgens, M. (2003) Sensitivity analysis for the assessment of the causal vaccine effects on viral load in HIV vaccine trials. Biometrics, 59, 531-41.

Heller, R., Rosenbaum, P.R. and Small, D.S. (2009) Split samples and design sensitivity in observational studies. J. Am. Statist. Assoc., 104, 1090-1101.

Hosman, C. A., Hansen, B. B. and Holland, P. W. H. (2010) The sensitivity of linear regression coefficients' confidence limits to the omission of a confounder. Ann. Appl. Statist., 4, 849870.

Hsu, J. Y., Small, D. S. and Rosenbaum, P. R. (2013) Effect modification and design sensitivity in observational studies. J. Am. Statist. Assoc., 108, 135-148.

Hsu, J. Y., Zubizarreta, J. R., Small, D. S. and Rosenbaum, P. R. (2015) Strong control of the familywise error rate in observational studies that discover effect modification by exploratory methods. Biometrika, 102, 767-782.

Jogdeo, K. (1977) Association and probability inequalities. Ann. Statist., 5, 495-504.

Lehrer, S. F., Pohl, R. V., \& Song, K. (2015) Targeting Policies: Multiple Testing and Distributional Treatment Effects. http://post.queensu.ca/〜lehrers/temult.pdf

Liu, W., Kuramoto, J. and Stuart, E. (2013) Sensitivity analysis for unobserved confounding in nonexperimental prevention research. Prev. Sci., 14, 570-580.

Lu, X. and White, H. (2015) Testing for treatment dependence of effects of a continuous treatment. Econometric Theory, 31, 1016-1053.

Marcus, R., Peritz, E. and Gabriel, K. R. (1976) On closed testing procedures with special reference to ordered analysis of variance. Biometrika, 63, 655-60. 
Neyman, J. (1923, 1990) On the application of probability theory to agricultural experiments. Statist. Sci., 5, 463-480.

Rosenbaum, P. R. (2002a) Observational Studies (2 ${ }^{\text {nd }}$ edition) New York: Springer.

Rosenbaum, P. R. (2002b) Attributing effects to treatment in matched observational studies. J. Am. Statist. Assoc., 97, 183-192.

Rosenbaum, P. R. (2004) Design sensitivity in observational studies. Biometrika, 91, 153-164.

Rosenbaum, P. R. (2005) Heterogeneity and causality: unit heterogeneity and design sensitivity in observational studies. Am. Statist., 59, 147-152.

Rosenbaum, P. R. and Silber, J. H. (2009) Amplification of sensitivity analysis in observational studies. J. Am. Statist. Assoc., 104, 1398-1405. (amplify function in the R package sensitivitymv)

Rubin, D. B. (1974) Estimating causal effects of treatments in randomized and nonrandomized studies. J. Educ. Psych., 66, 688-701.

Schapire, R.E., Freund, Y., Bartlett, P. and Lee, W.S. (1998) Boosting the margin: a new explanation for the effectiveness of voting methods. Ann. Statist., 26, 1651-1686.

Silber, J. H., Rosenbaum, P. R., McHugh, M. D., Ludwig, J. M., Smith, H. L., Niknam, B. A., Even-Shoshan, O., Fleisher, L. A., Kelz, R. R. and Aiken, L. H. (2016) Comparison of the value of better nursing work environments across different levels of patient risk. JAMA Surgery, 151, 527-536.

Stuart, E. A. and Hanna, D. B. (2013) Should epidemiologists be more sensitive to design sensitivity?" Epidemiol., 24, 88-89.

Wager, S. and Athey, S. (2015) Estimation and inference of heterogeneous treatment effects using random forests. arXiv preprint arXiv:1510.04342.

Zaykin, D. V., Zhivotovsky, L. A., Westfall, P. H., and Weir, B. S. (2002) Trucated product method of combining $P$-values. Genet. Epidemiol., 22, 170-185. (truncatedP function in the $R$ package sensitivitymv)

Zhang, H. and Singer, B. H. (2010) Recursive Partitioning and Applications. New York: Springer.

Zubizarreta, J. R., Cerdá, M. and Rosenbaum, P. R. (2013) Effect of the 2010 Chilean earthquake on posttraumatic stress: Reducing sensitivity to unmeasured bias through study design. Epidemiol., 24, 79-87. 
Table 1. Grouping of procedure clusters, with and without congestive heart failure (CHF).

\begin{tabular}{|c|c|c|c|c|c|}
\hline & Procedure Cluster & $\begin{array}{c}\text { No CHF } \\
\text { proc1 }\end{array}$ & $\begin{array}{l}\text { CHF } \\
\text { proc3 }\end{array}$ & $\begin{array}{c}\text { No } \mathrm{CHF} \\
\text { proc2 }\end{array}$ & $\begin{array}{l}\text { CHF } \\
\text { proc4 }\end{array}$ \\
\hline 1 & Adrenal procedures & $\mathrm{x}$ & $\mathrm{x}$ & & \\
\hline 2 & Appendectomy & $\mathrm{x}$ & & & $\mathrm{x}$ \\
\hline 3 & Bowel anastamoses & & & $\mathrm{x}$ & $\mathrm{x}$ \\
\hline 4 & Bowel procedures, other & & & $\mathrm{x}$ & $\mathrm{x}$ \\
\hline 5 & Breast procedures & $\mathrm{x}$ & $\mathrm{x}$ & & \\
\hline 6 & Esophageal procedures & & $\mathrm{x}$ & $\mathrm{x}$ & \\
\hline 7 & Femoral hernia procedures & $\mathrm{x}$ & $\mathrm{x}$ & & \\
\hline 8 & Gallbladder procedures & $\mathrm{x}$ & $\mathrm{x}$ & & \\
\hline 9 & Incisional and abdominal hernias & $\mathrm{x}$ & $\mathrm{x}$ & & \\
\hline 10 & Inguinal hernia procedures & $\mathrm{x}$ & $\mathrm{x}$ & & \\
\hline 11 & Large bowel resection & & & $\mathrm{x}$ & $\mathrm{x}$ \\
\hline 12 & Liver procedures & $\mathrm{x}$ & & & $\mathrm{x}$ \\
\hline 13 & Lysis of adhesions & & & $\mathrm{x}$ & $\mathrm{x}$ \\
\hline 14 & Ostomy procedures & & & $\mathrm{x}$ & $\mathrm{x}$ \\
\hline 15 & Pancreatic procedures & & $\mathrm{x}$ & $\mathrm{x}$ & \\
\hline 16 & Parathyroidectomy & $\mathrm{x}$ & $\mathrm{x}$ & & \\
\hline 17 & PD access procedure & & & $\mathrm{x}$ & $\mathrm{x}$ \\
\hline 18 & Rectal procedures & $\mathrm{x}$ & $\mathrm{x}$ & & \\
\hline 19 & Repair of vaginal fistulas & $\mathrm{x}$ & $\mathrm{x}$ & & \\
\hline 20 & Small bowel resection & & & $\mathrm{x}$ & $\mathrm{x}$ \\
\hline 21 & Splenectomy & & & $\mathrm{x}$ & $\mathrm{x}$ \\
\hline 22 & Stomach procedures & & & $\mathrm{x}$ & $\mathrm{x}$ \\
\hline 23 & Thyroid procedures & $\mathrm{x}$ & $\mathrm{x}$ & & \\
\hline 24 & Ulcer surgery & & & $\mathrm{x}$ & $\mathrm{x}$ \\
\hline 25 & Umbilical hernia procedures & $\mathrm{x}$ & & & $\mathrm{x}$ \\
\hline 26 & Ventral hernia repair & $\mathrm{x}$ & $\mathrm{x}$ & & \\
\hline
\end{tabular}


Table 2. Mortality in 23,715 matched pairs of a patient receiving surgery at a magnet hospital or a control hospital, where the pairs have been divided into five groups selected by CART.

\begin{tabular}{|c|c|c|c|c|c|c|}
\hline & \multicolumn{5}{|c|}{ Subgroups } & \multirow[t]{2}{*}{ Pooled } \\
\hline & Group 1 & Group 2 & Group 3 & Group 4 & Group5 & \\
\hline $\mathrm{CHF}$ & no & no & no & yes & yes & \\
\hline Procedures & proc1 & proc2 & proc2 & proc3 & proc4 & \\
\hline ER admission & both & no & yes & both & both & \\
\hline Number of Pairs & 10127 & 5636 & 2943 & 2086 & 2923 & 23715 \\
\hline Discordant Pairs & 210 & 293 & 488 & 217 & 760 & 1968 \\
\hline Percent Discordant $\%$ & 2.1 & 5.2 & 16.6 & 10.4 & 26.0 & 8.3 \\
\hline Odds Ratio & 1.41 & 1.53 & 1.09 & 1.28 & 1.18 & 1.23 \\
\hline Morality \%, Magnet & 0.9 & 2.5 & 10.1 & 4.9 & 16.5 & 4.7 \\
\hline Morality \%, Control & 1.3 & 3.5 & 10.8 & 6.2 & 18.6 & 5.6 \\
\hline \multicolumn{7}{|c|}{ Sensitivity analysis: Upper bounds on $P$-values for various $\Gamma$} \\
\hline \multirow[t]{2}{*}{$\Gamma$} & \multicolumn{5}{|c|}{ Subgroups } & Truncated \\
\hline & Group 1 & Group 2 & Group 3 & Group 4 & Group 5 & Product \\
\hline 1.00 & 0.008 & 0.000 & 0.195 & 0.039 & 0.013 & 0.000 \\
\hline 1.05 & 0.019 & 0.001 & 0.374 & 0.080 & 0.062 & 0.000 \\
\hline 1.10 & 0.042 & 0.003 & 0.576 & 0.143 & 0.184 & 0.012 \\
\hline 1.15 & 0.079 & 0.010 & 0.753 & 0.230 & 0.386 & 0.032 \\
\hline 1.17 & 0.099 & 0.015 & 0.809 & 0.270 & 0.479 & 0.044 \\
\hline 1.20 & 0.135 & 0.025 & 0.875 & 0.335 & 0.616 & 0.163 \\
\hline
\end{tabular}


Table 3. Use of the intensive care unit (ICU) in 23,715 matched pairs of a patient receiving surgery at a magnet hospital or a control hospital, where the pairs have been divided into five groups indicated in Figure 1.

\begin{tabular}{c|ccccc|c}
\hline & \multicolumn{7}{|c|}{ Subgroups } & Pooled \\
& Group 1 & Group 2 & Group 3 & Group 4 & Group5 & \\
\hline CHF & no & no & no & yes & yes & \\
Procedures & proc1 & proc2 & proc2 & proc3 & proc4 & \\
ER admission & both & no & yes & both & both & \\
\hline Number of Pairs & 10127 & 5636 & 2943 & 2086 & 2923 & 23715 \\
Discordant Pairs & 2675 & 2361 & 1282 & 859 & 970 & 8147 \\
Percent Discordant \% & 26.4 & 41.9 & 43.6 & 41.2 & 33.2 & 34.4 \\
Odds ratio & 1.63 & 2.05 & 1.67 & 1.70 & 1.88 & 1.78 \\
ICU \%, Magnet & 15.3 & 28.9 & 53.8 & 41.0 & 69.8 & 32.3 \\
ICU \%, Control & 21.7 & 43.3 & 64.6 & 51.7 & 80.0 & 42.0 \\
\hline
\end{tabular}

\begin{tabular}{c|cccccc|c}
\hline \multicolumn{7}{c}{ Sensitivity analysis: Upper bounds on $P$-values for various $\Gamma$} & \\
\hline$\Gamma$ & \multicolumn{7}{c}{ Subgroups } \\
& Group 1 & Group 2 & Group 3 & Group 4 & Group 5 & Product \\
\hline 1 & 0.000 & 0.000 & 0.000 & 0.000 & 0.000 & 0.000 \\
1.5 & 0.017 & 0.000 & 0.037 & 0.040 & 0.000 & 0.000 \\
1.6 & 0.312 & 0.000 & 0.254 & 0.203 & 0.009 & 0.000 \\
1.7 & 0.849 & 0.000 & 0.651 & 0.511 & 0.074 & 0.000 \\
1.8 & 0.993 & 0.002 & 0.916 & 0.798 & 0.276 & 0.049 \\
1.9 & 1.000 & 0.047 & 0.989 & 0.945 & 0.582 & 0.235 \\
\hline
\end{tabular}

Table 4. Mortality and ICU use in 5,636 pairs in Group 2. The table counts pairs of patients, not individual patients.

\begin{tabular}{r|rrr|r}
\hline Control Hospital & \multicolumn{3}{|c|}{ Magnet Hospital } & \\
\hline & Dead & Alive, ICU & Alive, no ICU & Total \\
\hline Dead & 23 & 72 & 105 & 200 \\
Alive, ICU & 60 & 744 & 1493 & 2297 \\
Alive, no ICU & 56 & 726 & 2357 & 3139 \\
\hline Total & 139 & 1542 & 3955 & 5636 \\
\hline
\end{tabular}




\section{Mortality in 23715 Matched Pairs}

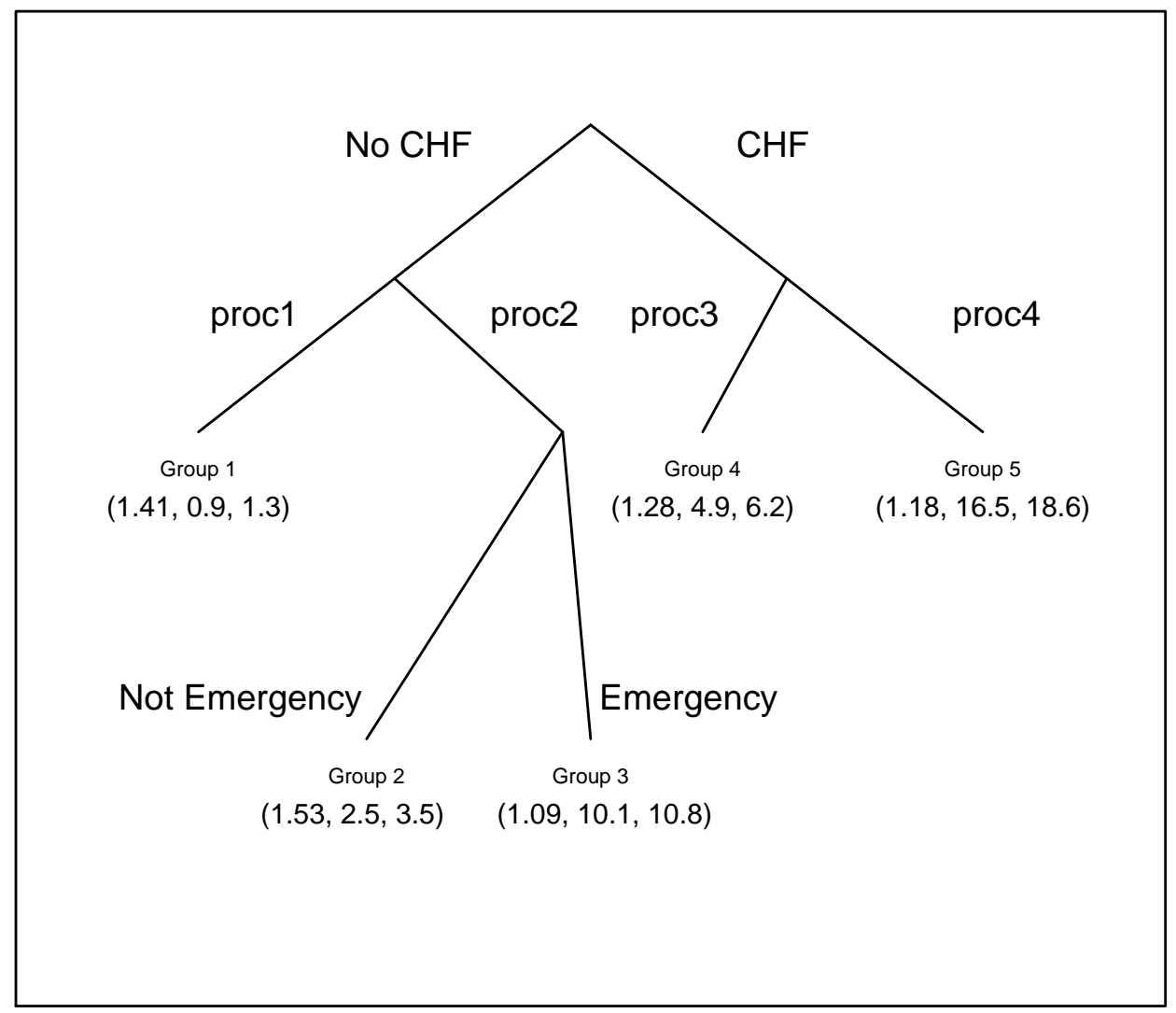

Fig. 1. Mortality in 23,715 matched pairs of two Medicare patients, one receiving surgery at a magnet hospital identified for superior nursing, the other undergoing the same surgical procedure at a conventional control hospital. The three values $(A, B, C)$ at the nodes of the tree are: $A=$ McNemar odds ratio for mortality, control/magnet, $\mathrm{B}=30$-day mortality rate $(\%)$ at the magnet hospitals, $\mathrm{C}=30$-day mortality rate $(\%)$ at the control hospitals. 